Meta

Journal des traducteurs

Translators' Journal

\title{
La bi-textualité : vers une nouvelle génération d'aides à la traduction et la terminologie
}

\section{Pierre Isabelle}

Volume 37, numéro 4, décembre 1992

Études et recherches en traductique / Studies and Researches in Machine Translation

URI : https://id.erudit.org/iderudit/003228ar

DOI : https://doi.org/10.7202/003228ar

Aller au sommaire du numéro

Éditeur(s)

Les Presses de l'Université de Montréal

ISSN

0026-0452 (imprimé)

1492-1421 (numérique)

Découvrir la revue

Citer cet article

Isabelle, P. (1992). La bi-textualité : vers une nouvelle génération d'aides à la traduction et la terminologie. Meta, 37(4), 721-737.

https://doi.org/10.7202/003228ar
Résumé de l'article

Les postes de travail de traducteur actuels se concentrent encore trop peu sur les aspects proprement traductionnels de la tâche du traducteur. Nous montrons que le concept de bi-texte permet d'envisager de nouvelles possibilités à cet égard. Un bi-texte consiste en un couple de textes (une source et sa traduction) unis par une représentation explicite de leurs correspondances traductionnelles. Structurée en bi-texte, la production d'un service de traduction devient une véritable mémoire d'entreprise , dont les richesses peuvent être exploitées à l'aide d'outils comme le concordancier bilingue . Le concept de bi-texte permet également d'envisager le développement d'outils de critique de traductions, capables par exemple de détecter la présence de faux amis dans une traduction. Nous disposons des algorithmes requis pour calculer automatiquement une représentation bi-textuelle (partielle), à partir d'un texte et de sa traduction. Nous en sommes à l'étape de maquettage d'outils de concordance bilingue et de critique de traductions.
Ce document est protégé par la loi sur le droit d'auteur. L'utilisation des services d’Érudit (y compris la reproduction) est assujettie à sa politique d'utilisation que vous pouvez consulter en ligne.

https://apropos.erudit.org/fr/usagers/politique-dutilisation/ 


\title{
LA BI-TEXTUALITÉ: VERS UNE NOUVELLE GÉNÉRATION D'AIDES À LA TRADUCTION ET LA TERMINOLOGIE'
}

\author{
PIERRE ISABELLE \\ Centre canadien de recherche sur \\ linformatisation du travail, Laval, Canada
}

\begin{abstract}
Résumé
Les postes de travail de traducteur actuels se concentrent encore trop peu sur les aspects proprement traductionnels de la tâche du traducteur. Nous montrons que le concept de bi-texte permet d"envisager de nouvelles possibilités à cet égard. Un bi-texte consiste en un couple de textes (une source et sa traduction) unis par une représentation explicite de leurs correspondances traductionnelles. Structurée en bi-texte, la production d'un service de traduction devient une véritable mémoire d'entreprise, dont les richesses peuvent être exploitées à l'aide d'outils comme le concordancier bilingue. Le concept de bi-texte permet également d'envisager le développement d'outils de critique de traductions, capables par exemple de détecter la présence de faux amis dans une traduction. Nous disposons des algorithmes requis pour calculer automatiquement une représentation bi-textuelle (partielle), à partir d'un texte et de sa traduction. Nous en sommes à l'étape de maquettage d'outils de concordance bilingue et de critique de traductions.
\end{abstract}

\begin{abstract}
Existing translator's workstations still provide lery little support for the central and defining aspects of the translator's task. The concept of bi-text is shown to open altogether new possibilities for the workstation. A bi-text is a pair of texts (a source and its translation) linked by a set of explicit translational correspondences. If translations are organized bitextually, the production of a translation service can become a veritable corporate memory. whose riches can be tapped by tools like bilingual concordancing systems. Bi-textual representations also provide the foundation for translation critiquing tools, capable for example of detecting the presence of deceptive cognates in a translation. Algorithms have already been developed that can automatically produce a (partial) bi-textual representation for a text and its translation. We are currently in the process of developing prototype concordancing and translation critiquing tools.
\end{abstract}

\section{CONTEXTE : LE PROGRAMME DU CCRIT EN TRADUCTION ASSISTÉE PAR ORDINATEUR}

Depuis 1986, le CCRIT (Communications Canada) poursuit un programme de recherche appliquée en traduction assistée par ordinateur. Ce programme vise à aider les traducteurs canadiens à faire face à une demande qui croît beaucoup plus vite que les ressources disponibles pour la combler.

Depuis le début des années 50 , beaucoup d'efforts on visé l'avènement de la machine à traduire universelle (MTU): une machine capable de produire des traductions de haute qualité, de façon entièrement automatique, pour n'importe quel genre de texte. Malheureusement, ces tentatives ont connu très peu de succès. Sur le marché actuel de la traduction, la traduction automatique (TA) demeure au mieux un phénomène marginal. La plupart des services de traduction considèrent que, dans l'état actuel de l'art, ils ne peuvent tirer aucun profit de la TA. 
Trop de gens encore ne réalisent pas à quel point la TA est un problème difficile. Elle vise rien de moins que l'automatisation de l'une des manifestations les plus complexes de l'intelligence humaine. La solution des problèmes de traduction exige non seulement une connaissance intime de deux systèmes linguistiques qui sont chacun d'une complexité inouïe, mais également la capacité de raisonner intelligemment à propos de l'univers extralinguistique dans lequel sont interprétés les textes. Bref, la MTU relève de l'intelligence artificielle universelle, et pour longtemps encore, de la science-fiction.

Le programme du CCRIT prend ce constat comme point de départ, et cherche des moyens de tirer parti des technologies bien moins qu'optimales qui existent à l'heure actuelle. En pratique, ceci signifie la recherche de compromis acceptables sur l'un ou plusieurs des attributs de la MUT: qualité des résultats, automatisation totale, généralité.

D'emblée, nous excluons les compromis relatifs à la qualité des traductions. D'une part, les consommateurs du marché canadien exigent, pour la plupart, des traductions de haute qualité. D'autre part, l'expérience a démontré qu'il est rarement rentable de réviser manuellement les traductions médiocres que produisent invariablement les systèmes qui tentent le diable, en visant simultanément une automatisation poussée et une applicabilité générale. Il nous faut plutôt nous tourner vers des compromis relatifs à la généralité des systèmes ou à leur niveau d'automatisation. Dans chacun des deux cas, nous croyons qu'il est possible de développer des stratégies gagnantes, et c'est exactement ce que le programme du CCRIT s'emploie à démontrer.

Un premier volet de ce programme vise le développement de systèmes de TA spécialisés. Ce volet puise son inspiration dans les travaux du groupe TAUM de l'Université de Montréal qui, dès 1976, a démontré que la TA pouvait être très rentable dans le cas de «souslangues» restreintes, comme celle des prévisions météorologiques. Les activités du CCRIT dans ce domaine ont fait l'objet de plusieurs publications, comme Isabelle et al. 1988, Dymetman et al. 1990, que le lecteur est invité à consulter. Le présent document est plutôt consacré à l'autre volet du programme, celui de la traduction assistée par ordinateur (TAO).

Le terme traduction assistée par ordinateur est passablement galvaudé depuis quelques années. Trop souvent, il est utilisé comme euphémisme pour des systèmes de traduction automatique de mauvaise qualité, qui tendent à produire des résultats jugés irrécupérables par les traducteurs. La vraie traduction assistée ne fait pas du traducteur un concierge de sorties machine exécrables. La machine n'y fait que ce qu'elle sait bien faire. En principe, on peut envisager deux variantes de la TAO: la traduction machine assistée par l'humain (TMAH) et la traduction humaine assistée par ordinateur (THAM).

Dans la TMAH, la machine prend l'initiative du processus et sollicite l'aide d'un humain pour les problèmes qu'elle se juge incapable de résoudre. Malheureusement, pour des textes généraux, il y a tellement de problèmes que la machine s'avère incapable de résoudre que la TMAH n'a jusqu'à maintenant donné lieu qu'à des systèmes pervers: dans lesquels une machine idiote tend à subordonner un humain qui sait infiniment mieux faire qu'elle.

Par contraste, dans la THAM, l'humain conserve toujours l'initiative du processus. Il assume la responsabilité de l'opération centrale, à savoir la reformulation du texte de départ en langue d'arrivée, et à son gré, il fait appel à la machine pour l'aider dans les aspects les plus mécaniques de sa tâche. Le concept de poste de travail de traducteur (PTT) s'inscrit dans cette perspective. Le PTT se borne à fournir un appui à la démarche naturelle du traducteur, en lui offrant le recours à toute une gamme d'aides informatisées: traitement de textes, vérification d'orthographe, dictionnaires électroniques, bases de données terminologiques, etc.

Comme l'automatisation est très partielle, les gains de productivité vont évidemment demeurer limités. Mais il s'agira au moins de gains réels. Et loin de compromettre 
la qualité des traductions, on contribue à l'augmenter en misant sur la machine pour ce qu'elle sait bien faire. Aussi, cette approche a le mérite d'être applicable à n'importe quel genre de textes. La THAM, telle qu'incarnée dans un PTT, constitue une stratégie gagnante.

Le programme du CCRIT a déjà produit plusieurs prototypes de postes de travail de traducteurs (Macklovitch 1989, 1991). À ce jour, les essais se sont avérés concluants. Le concept de PTT est là pour rester, et fait peu à peu son chemin dans la communauté des traducteurs.

\section{LES LIMITES DES OUTILS D'AIDE EXISTANTS}

Reste que les postes de travail existants font encore trop peu pour le traducteur. La traduction est une tâche complexe. S'il n'y a pas unanimité quant à la meilleure façon de décomposer cette activité en sous-tâches, le découpage proposé par Socioscope 1986 est assez typique de ceux que l'on trouve dans la littérature pertinente:

1) réception, planification, etc.
2) première lecture
3) lecture approfondie
4) recherche
5) transfert (composition mentale)

6) rédaction

7) première révision

8) seconde révision

9) lecture d'épreuve

10) livraison, facturation, etc.

\section{Les tâches du traducteur d'après Socioscope}

Un examen des PTT actuels révèle qu'ils n'accordent pas une attention égale à chacune de ces sous-tâches. Et, ce qui est peut-être plus surprenant, c'est que les tâches les mieux couvertes ne sont justement pas celles qui sont les plus spécifiques au problème de la traduction. Par exemple, les outils présents dans la version 2.0 du PTT du CCRIT (Macklovitch 1991) se répartissent de la manière suivante.

Les étapes 1,2 et 3 concernent le traducteur en sa qualité de lecteur, de consommateur de textes. À l'étape 1, le traducteur peut au besoin recourir à un programme de conversion de formats de fichiers pour saisir sur son PTT un texte source autrement incompatible. Ensuite, si le traducteur sait qu'il a affaire à une mise à jour d'un texte déjà traduit, et s'il peut retrouver la version précédente (sur support ordinolingue), il pourra utiliser un comparateur de fichiers pour limiter sa tâche aux segments qui ont été modifiés. Enfin, il pourra utiliser un compte-mots pour évaluer et planifier sa tâche. Pour l'étape 2, il est laissé à luimême. Mais, pour «approfondir» sa lecture, à l'étape 3, il pourra consulter des dictionnaires de la langue source, et même se servir d'un logiciel concordancier pour décortiquer le texte.

Les étapes 4 et 5 constituent le cœur du travail traductionnel : c'est là que sont déterminées les équivalences entre les langues source et cible. Les outils pertinents offerts sur le PTT se résument à ceci : dictionnaires bilingues et banques de terminologie informatisées. L'apport de ces outils, sans être négligeable, $n$ 'a rien d'extrêmement spectaculaire par rapport aux traditionnels imprimés. Au delà des aides lexicographiques et terminologiques, la reformulation traductionnelle, le «transfert», s'effectue de façon purement manuelle.

Comme tout autre langagier, le traducteur se servira d'un traitement de texte ou d'un système d'éditique pour la portion rédactionnelle de sa tâche (étape 6). Pour la lecture d'épreuves (étape 9), il pourra recourir à un vérificateur d'orthographe (et souvent aussi à un vérificateur grammatical). Quant à la révision dans ses aspects proprement traductionnels, à savoir la vérification des équivalences établies (étapes 7 et 8), le PTT n'a pour l'instant pas grand-chose à offrir au traducteur. 
Dans la mesure où le PTT 2.0 du CCRIT est assez typique des environnements qui sont présentement offerts aux traducteurs, il ne paraît pas exagéré de dire qu'il reste encore beaucoup de chemin à faire avant de pouvoir prétendre offrir un support intégral à l'activité traduisante.

Les outils d'aides à la lecture, à la rédaction, à la gestion et à la transmission de textes vont continuer de progresser indépendamment de la communauté des traducteurs, car ils concernent un public beaucoup plus large. Le traducteur bénéficiera de ces progrès, notamment par: a) de meilleures interfaces utilisateur; b) un courrier électronique fiable permettant un échange facile des textes source et leurs traductions; c) de meilleurs outils de vérification d'orthographe et de grammaire; et d) un peu plus tard, d'outils de dictée vocale.

Toutefois, en ce qui concerne le coeur de la tâche du traducteur, si progrès il y a, ils devront venir de la recherche en traductique. Au delà des lexiques informatisés, et en deçà de la traduction automatique, comment faire pour améliorer le niveau de support à l'activité traduisante? De toute évidence, nous avons besoin de nous tourner vers de nouveaux concepts.

Dans le reste du présent document, nous allons arguer que le concept de bi-texte est précisément ce dont nous avons besoin, et que ce concept ouvre la voie à rien de moins qu'une nouvelle génération d'aides informatisées à la traduction.

\section{LE CONCEPT DE BI-TEXTE}

Qu'est-ce qui distingue les traducteurs, en tant que groupe, des autres langagiers? La réponse est évidente. Par définition, les traducteurs opèrent non pas sur un mais bien sur deux textes: l'un pré-existant dans une langue source et l'autre à produire dans une langue différente, en respectant une certaine relation d'équivalence avec le premier. Le maintien de cette relation d'équivalence est le pivot de l'activité traduisante. Ce n'est que sur une caractérisation de cette relation que l'on pourra fonder des aides proprement traductionnelles.

Il sera utile, dans cette perspective, de réexaminer certaines propriétés générales des équivalences traductionnelles. Rappelons d'abord que la relation d'équivalence traductionnelle a pour domaine des ensembles infinis, puisque l'ensemble des textes possibles dans chaque langue est infini. Une caractérisation finie de cette relation doit par conséquent faire intervenir des mécanismes récursifs. En pratique, ceci signifie que la relation doit se fonder sur un ensemble fini d'équivalences élémentaires ${ }^{2}$, et un ensemble fini de lois de combinaison de ces équivalences élémentaires. Une conséquence importante de cette nécessité, c'est que les traductions obéissent à un principe dit de compositionnalité: la traduction d'un segment complexe est généralement une fonction de la traduction de ses parties, et ce, jusqu'au niveau d'un ensemble d'unités élémentaires.

Ce principe de compositionnalité fait écho à une intuition évidente: lorsque deux textes sont en relation de traduction, on peut généralement observer des correspondances entre les portions respectives de ces deux textes. Étant donné un segment quelconque $S 2$ du texte d'arrivée, on peut généralement lui faire correspondre un segment $S 1$ de manière que $S 2$ constitue la traduction de $S 1$. Comme le remarque Harris 1988 , certains modes de mise en page souvent privilégiés pour les traductions visent précisément à mettre en évidence certaines de ces correspondances. Par exemple, la mise en page «côte-à-côte» cherche à coupler les paragraphes, alors que la mise en page «interlinéaire» couple des unités plus petites, comme des phrases.

Harris 1988, 1988 propose le terme bi-texte pour désigner un couplage explicite des segments d'un texte source avec ceux de sa traduction. Nous adoptons ce terme, en lui donnant la définition technique suivante. Formellement, un bi-texte est un quadruplet 
$<T_{1}, T_{2}, F s, C>$ dans lequel $T_{1}$ et $T_{2}$ sont deux textes, $F s$ est une fonction qui décompose ces textes en des ensembles de segments, et $C$ est un ensemble de correspondances entre $F s\left(T_{1}\right)$ et $F s\left(T_{2}\right)$.

Cette définition soulève un certain nombre de questions importantes. Premièrement, quelle est la nature des segments (produits par la fonction $F s$ ) qui sont mis en correspondance? Si les mises en page côte à côte ou interlinéaires semblent supposer une simple séquence de segments non structurés, le principe de compositionnalité suggère plutôt un système de correspondances hiérarchisées allant d'unités textuelles très larges vers des unités de plus en plus petites. Une façon très naturelle d'obtenir un tel système hiérarchique consiste à assimiler la fonction de segmentation $F s$ à une fonction d'analyse syntaxique. On obtient alors pour les textes source et cible une décomposition qui part des unités les plus larges (sections, paragraphes, etc.) et descend jusqu'aux mots (voire jusqu'aux morphèmes). Une telle décomposition permet la formulation de correspondances hiérarchisées entre les deux textes, comme dans le schéma de la Figure 1.

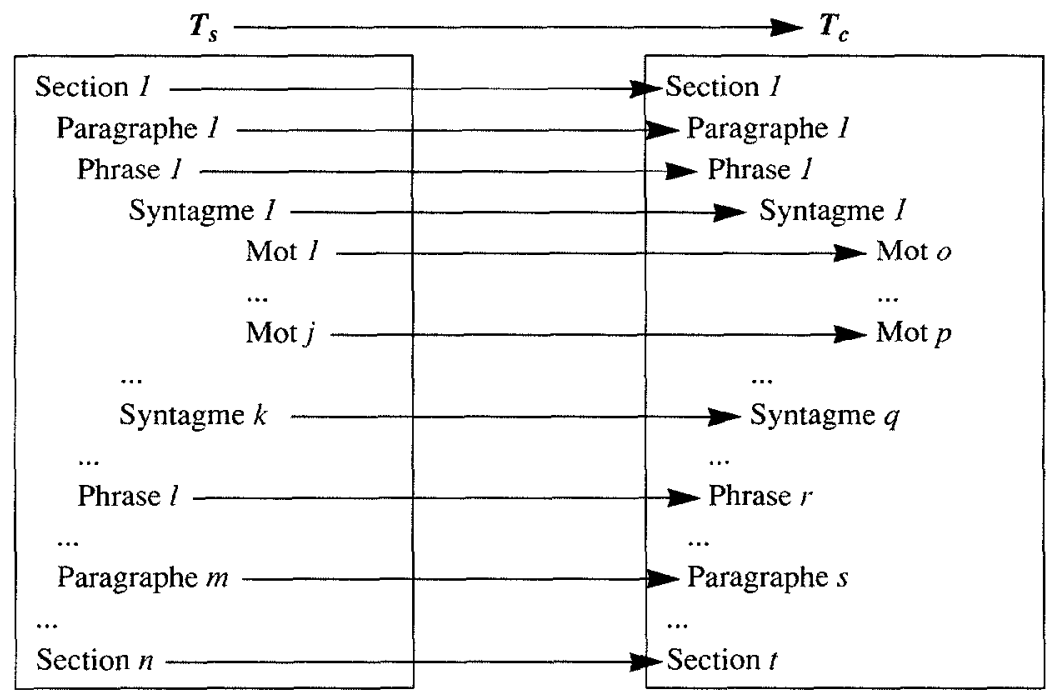

Figure 1:

Correspondances hiérarchisées entre un texte source et sa traduction

Toutefois, il est évident qu'une caractérisation adéquate doit permettre des correspondances plus complexes que le un-pour-un systématique qui apparaît dans la Figure 1 . Un exemple aussi banal que celui de la traduction de potato par pomme de terre met en jeu une correspondance d'un mot à trois mots. Le modèle hiérarchique facilite la formulation des correspondances de ce genre: on peut y décrire de façon naturelle une correspondance entre des unités de rang différent. L'unité de rang mot potato correspond à l'unité de rang syntagme pomme de terre.

D'autre part, si l'on prend les «structures syntaxiques superficielles» comme base représentationnelle pour la formulation des correspondances, on rencontre certaines difficultés, comme celle des constituants discontinus. Par exemple, il sera difficile de décrire la correspondance qui s'établit entre not et ne...pas dans les exemples de (E1) ou entre 
turn...off et fermer dans ceux de (E2), parce que l'un des termes de la relation ne forme pas une unité identifiée comme telle dans la représentation:

(E1) a) Max has not seen Conrad.

b) Max n'a pas vu Conrad.

(E2) a) Max turned the radio on.

b) Max alluma la radio.

La conclusion qui s'impose est la suivante. Pour produire un bi-texte qui rend explicites toutes les correspondances traductionnelles, il faudrait faire appel à une fonction de segmentation très abstraite, qui produirait des «représentations syntaxiques profondes», ou des «représentations sémantiques», voire des «représentations conceptuelles». Globalement, il s'agit donc d'un problème difficile.

Il n'en demeure pas moins que le problème du calcul automatique de bi-textes est beaucoup moins difficile que celui de la TA, et ce pour au moins deux raisons.

Premièrement, le calcul de bi-textes suppose une compétence passive, par opposition à la compétence active mise en jeu par la TA. On peut comparer le problème de reconstruction d'un bi-texte à la situation d'un traducteur novice qui, sans être capable de produire lui-même une bonne traduction, est quand même en mesure de comprendre le travail d'un traducteur expert. Une compétence passive est toujours bien plus facile à acquérir qu'une compétence active.

Deuxièmement, le modèle hiérarchisé des correspondances traductionnelles implique la possibilité de faire varier un paramètre de résolution dans la spécification des correspondances. Un bi-texte à basse résolution se contentera d'énoncer des correspondances entre les unités de rang supérieur, comme les paragraphes ou les phrases. Un bi-texte à haute résolution montrera en plus les correspondances entre les unités de rang inférieur: syntagmes, mots, voire morphèmes. Cette notion de résolution n'a pas d'homologue en TA: on ne saurait traduire un paragraphe sans traduire du même coup tous ses éléments.

La qualité d'un bi-texte s'évalue donc en fonction de deux critères: son niveau de résolution (les correspondances se font-elles à un niveau assez fin?), et son niveau de précision (quelle est la proportion de correspondances correctes?). Or, comme nous le verrons à la section 6., des efforts très modestes ${ }^{3}$ ont déjà abouti au développement d'algorithmes capables de créer automatiquement des bi-textes d'un niveau de résolution limité (typiquement, le niveau des phrases), mais d'un niveau de précision très élevé.

Dans les deux prochaines sections, nous allons tenter de démontrer que la disponibilité de bi-textes permet d'envisager de nouvelles formes très concrètes d'aide à la traduction.

\section{UNE MÉMOIRE D'ENTREPRISE POUR LES TRADUCTEURS}

La plupart des traducteurs sont quotidiennement confrontés à des problèmes de traduction dont la solution peut s'avérer laborieuse. Les outils présentement à leur disposition, qu'ils s'agisse de dictionnaires de langue, de dictionnaires bilingues, de lexiques spécialisés ou de banques de terminologie, ont certes beaucoup de mérites. Toutefois, ces outils, malgré leurs coûts de développement élevés, demeurent toujours très incomplets. C'est là une source de frustration constante pour le traducteur.

Au Canada seulement, bon an mal an, le volume de traductions atteint au moins un demi-milliard de mots. Il faut se rendre à l'évidence. La masse des traductions produites chaque année contient infiniment plus de solutions à plus de problèmes que tous les outils de référence existants et imaginables! Si seulement les services de traduction pouvaient considérer que ces masses de textes qu'ils génèrent constituent une richesse qu'ils pourront réexploiter... 
Malheureusement, ce n'est pas du tout le cas présentement. Le service de traduction typique n'est même pas en mesure de garantir que l'un de ses traducteurs n'est pas en train de refaire à partir de zéro une traduction déjà faite par un autre. L'archivage des traductions demeure la plupart du temps peu systématique, artisanal, sinon carrément aléatoire. Les archives ordinolingues, dans les rares cas où elles existent, ne sont d'à peu près aucune utilité, faute d'outils capables d'aider le traducteur à en exploiter le potentiel. Dans ces conditions, comment ne pas penser que le traducteur moyen perd beaucoup de son temps à réinventer (ou pire, à rater) des solutions que ses collègues (voire lui-même) avaient déjà trouvées?

Supposez un instant qu'un service de traduction reçoive tous ses textes sources sur support ordinolingue. Supposez qu'une fois le travail terminé, on archive systématiquement, côte à côte, le texte source et sa traduction (les problèmes de compatibilité de fichiers informatiques ayant été résolus). Supposez enfin que, par un moyen quelconque, ces archives puissent être converties en une masse de bi-textes. Cela fait, il est vrai, beaucoup de suppositions. Mais ce que l'on obtient ainsi en vaut bien la peine: il s'agit de rien de moins qu'une véritable mémoire d'entreprise exploitable !

Dans cette masse de bi-texte, chaque segment de l'une des langues est lié à sa traduction dans l'autre langue. Par conséquent, chaque segment qui constitue un problème de traduction est lié à un segment solution. Le bi-texte constitue de ce fait l'amorce d'une approche à base mémorielle: au lieu de recréer à chaque fois une solution à un problème de traduction particulier, on se donne la possibilité de rappeler des solutions déjà trouvées.

Comment faire en pratique? Plusieurs approches sont envisageables. La plus ambitieuse s'intéresse à la possibilité de mettre au point des systèmes de traduction automatique à base mémorielle (par opposition aux systèmes à base de règles qui existent à 1'heure actuelle). De tels systèmes devront être capables de généraliser à partir d'exemples (voir Sato et Nagao 1990). Pour le moment, on ne peut parler ici que d'une avenue de recherche intéressante.

Une approche moins ambitieuse vise le développement de systèmes capables de rappeler la traduction de segments (quasi) identiques à ceux que l'on s'apprête à traduire, et d'en faire un matériau utilisable pour le traducteur. Certains systèmes commerciaux existants ${ }^{4}$ incorporent déjà quelques éléments de cette approche, qui nous paraît prometteuse à moyen terme.

Enfin, une solution encore moins ambitieuse et probablement plus universellement utile, consiste à fournir au traducteur le moyen d'interroger sélectivement et sur demande une base de données bi-textuelles. Confronté à un problème de traduction spécifique, le traducteur jugera lui-même de l'opportunité d'une recherche dans la mémoire d'entreprise. Pour ce faire, il aura accès à un outil relativement simple, mais tout à fait nouveau et qui présente à notre avis un intérêt majeur: le concordancier bilingue.

Une concordance est un recensement systématique de toutes les occurrences d'un mot ou d'une suite de mots avec leurs contextes d'apparition dans un texte. La concordance monolingue est un outil connu et apprécié depuis longtemps des langagiers, y compris de certains traducteurs et terminologues 5 . On trouvera en annexe un petit fragment d'une concordance monolingue sur le mot countervail dans le Journal des débats de la Chambre des Communes. La concordance permet de cerner rapidement l'emploi qui est fait dans les textes du mot ou de l'expression concernée.

Mais la concordance monolingue n'est pas spécifiquement orientée vers les besoins du traducteur. La concordance bilingue est au contraire un outil expressément orienté vers la recherche de solutions à des problèmes de traduction particuliers. Il s'agit tout simplement d'une concordance dans laquelle chaque contexte d'apparition d'un mot ou d'une expression est accompagné de sa traduction. 
Le lecteur trouvera également en annexe une version bilingue de la même concordance sur countervail. Un simple coup d'œil permet de constater que les traducteurs ont généralement opté pour une traduction par le terme droit compensateur, bien que certains semblent lui préférer droit compensatoire.

L'annexe contient également des concordances bilingues sur deux expressions de la langue générale: l'expression française cartes sur table et l'expression anglaise add insult to injury. Un examen attentif montre que si certaines traductions de ces expressions paraissent parfois ternes, il n'y pas besoin de chercher très longtemps pour découvrir des solutions surprenantes, inédites, parfaitement satisfaisantes, et parfaitement introuvables dans les dictionnaires. La mémoire d'entreprise d'un service de traduction est un trésor enfoui sous ses pieds!

\section{LA CRITIQUE DE TRADUCTIONS}

Bien entendu, les traductions ne sont pas toutes parfaites, et la mémoire d'entreprise doit être utilisée avec discernement. Sa fiabilité ne peut s'accroître que par une amélioration constante de la qualité des traductions du service. Or la notion de bi-texte a aussi certaines incidences sur la question du contrôle de la qualité des traductions. Il y a en effet tout lieu de penser que la structure bi-textuelle fournit le fondement requis pour mettre au point des outils capables d'effectuer des critiques de traductions.

Depuis quelques années, on voit apparaître sur le marché du logiciel des outils critiques pour la rédaction: détecteurs de fautes d'orthographe, de grammaire et même de style. Ces outils sont encore bien imparfaits, mais leur qualité s'améliore d'année en année, et de plus en plus de gens y ont recours pour la correction d'épreuves. Y compris, bien entendu, un certain nombre de traducteurs.

Ces outils d'aide à la rédaction ne s'intéressent aucunement au problème spécifique de la révision de traductions. Les erreurs de correspondance traductionnelle, même les plus grossières, leur sont inaccessibles. Et pour cause, ces outils ne savent examiner qu'un seul texte à la fois: la notion de bi-textualité leur est parfaitement étrangère.

Pensons par exemple à ces erreurs d'inattention qui font que l'on oublie (plus souvent qu'on pourrait le croire) de traduire des segments d'une taille considérable: une phrase, voire un paragraphe. Il n'existe présentement sur le marché aucun outil capable de lire un texte et sa traduction, et de relever automatiquement ce genre d'erreurs.

Effectuer une critique traductionnelle revient à vérifier que les correspondances constitutives d'une traduction particulière possèdent certaines propriétés spécifiques. Cette vérification devient possible précisément dans la mesure où les correspondances ont été rendues explicites, c'est-à-dire dans la mesure où l'on dispose de représentations bi-textuelles.

Les propriétés particulières que l'on pourra vérifier vont dépendre du niveau de résolution du bi-texte. Moyennant une résolution bi-textuelle très grossière, on devrait déjà pouvoir vérifier que le traducteur n'a pas omis de traduire des paragraphes, ou des phrases du texte de départ dans sa traduction. Il s'agit là de cas particuliers d'une propriété générale que l'on pourrait appeler l'exhaustivité d'une traduction.

Moyennant une augmentation graduelle du niveau de résolution, on pourra se tourner vers des propriétés peut-être plus intéressantes. Comme par exemple, celle d'être exempte d'interférences linguistiques en provenance de la langue source.

Prenons le problème classique des faux amis. Un faux ami «complet» est un couple de segments, qui constitue toujours une correspondance illicite, comme definitely/définitivement ${ }^{6}$. Un faux ami «partiel» est un couple de segments qui constitue une correspondance illicite dans certains contextes seulement (par exemple, camerałcaméra). Les analyses que nous avons effectuées sur des échantillons du Journal des débats de la 
Chambre des Communes montrent que le problème des faux amis est loin d'être marginal, et nous amènent à conclure que des aides seraient d'une très grande utilité 7 .

Un bi-texte dont la résolution atteint le niveau du mot et dont la précision est très élevée permettrait de garantir la détection automatique des faux amis «complets». Sans attendre cette situation idéale, nos analyses préliminaires tendent à indiquer que : a) même avec une résolution moindre, on peut obtenir un excellent rapport signal/bruit dans la détection des faux amis complets; et b) certaines techniques pourraient également permettre d'obtenir de bons résultats dans la détection d'une proportion importante de faux amis partiels.

On pourrait encore envisager la vérification automatique d'autres propriétés. Celle de la cohérence terminologique constitue un candidat possible. On imagine en ce cas un mécanisme capable d'explorer un bi-texte en y recherchant certaines classes de situations où un terme du texte de départ se voit associer plusieurs équivalents différents dans le texte d'arrivée.

Les travaux dans cette direction ne font que commencer. Mais, à court et à moyen terme, leur impact sur la grande communauté des traducteurs pourrait s'avérer beaucoup plus important que celui de la traduction automatique.

\section{CREATION AUTOMATIQUE DE BI-TEXTES}

Depuis le début des années 60, la recherche en traductique s'est surtout concentrée sur des méthodes dites «à base de règles» qui permettraient de faire de la traduction automatique. Malgré des progrès évidents, les résultats tant espérés se font toujours attendre. Récemment, un groupe de recherches d'IBM (Brown et al. 1990) a provoqué une commotion dans la communauté de $\mathrm{TA}$, en remettant au goût du jour une approche qui était tombée en défaveur avec l'émergence de la linguistique chomskyenne. Plus spécifiquement, il s'agit d'une approche statistique aux problèmes de traduction, qui fonctionne «à base de corpus» plutôt qu'à base de règles.

Le débat entre les approches classiques et les approches à base de corpus promet de faire rage pour quelque temps encore dans la communauté scientifique. En ce qui nous concerne, nous ne voyons nullement la statistique comme une panacée au problème de la TA.

Toutefois, les approches statistiques possèdent certaines caractéristiques qui leur confèrent des propriétés très différentes de celles des approches à base de règles. Brown et al. 1991 ainsi que Gale et Chruch 1991 ont montré que certaines méthodes statistiques permettaient de produire automatiquement un «alignement» des phrases d'un texte et sa traduction, et ce avec un très bon taux de réussite. Dans notre terminologie, nous disons que ces algorithmes permettent la création automatique de bi-textes dont le niveau de précision est élevé et dont le niveau de résolution est celui de la phrase.

S'il n'est pas du tout certain que des résultats de ce genre nous rapprochent beaucoup de la traduction automatique, ils représentent un développement d'une très grande importance en ce qui a trait aux aides à la traduction.

Des travaux en cours au CCRIT nous ont déjà permis de reproduire ces résultats: nous disposons présentement de programmes qui nous ont permis d'aligner les phrases d'un corpus bilingue constitué de 100 millions de mots du Journal des débats de la Chambre des Communes. Le laboratoire extraordinaire que constitue ce bi-texte gigantesque nous a permis d'amorcer des travaux sur un concordancier bilingue ainsi que sur la reconnaissance automatique des faux amis. Nous travaillons également à certaines extensions des algorithmes d'alignement qui devraient nous permettre d'améliorer leur niveau de précision (voir Simard, Foster et Isabelle à paraître) ainsi que leur niveau de résolution. Nous sommes confiants de pouvoir rapporter des résultats tangibles dans un proche avenir. 


\section{Notes}

1. Ce document est une version revue et augmentée d'une communication présentée au colloque Terminologie et documentation (Hull, octobre 1991), sous le titre «Une nouvelle génération d'aides à la traduction et la terminologie».

2. Par exemple, un dictionnaire bilingue. Bien entendu, on doit souvent admettre comme élémentaires des équivalences entre des unités linguistiques plus larges que le mot. C'est le cas des expressions idiomatiques, des proverbes, etc. Ce fait n'enlève rien à la force de notre argument.

3. Modestes en particulier lorsqu'on les compare aux efforts gigantesques qui ont été investis (ou engloutis) dans la traduction automatique depuis une quarantaine d'années.

4. Par exemple, les systèmes ALPS TSS, et UNITRAN.

5. Le PTT 2.0 du CCRIT comprend un concordancier monolingue.

6. Il y a forcément erreur dans ces cas, mais l'erreur n'en est pas forcément une de traduction. Il arrive en effet que ce soit l'auteur du texte source qui commette un faux ami et que le traducteur corrige cette erreur en traduisant l'intention plutôt que la lettre du texte source. Pour des raisons que nous ne pouvons expliquer ici, ce fait ne nous semble pas compromettre sérieusement la possibilité d'une détection des faux amis produits par le traducteur.

7. Nous n'entendons nullement ici critiquer les traducteurs eux-mêmes. L'erreur est humaine, et les contraintes temporelles et matérielles qui gouvernent chaque situation de traduction peuvent la rendre plus difficilement évitable.

\section{BIBLIOGRAPHIE}

BROWN, P., COCKE, J., DELLA PIETRA, S., DELLA PIETRA, V., JELINEK, F., LAFFERTY, J., MERCER, R. et P. ROOSIN (1990) : «A Statistical Approach to Machine Translation», Computational Linguistics, 16-2, pp. 79-85.

BROWN, P., LAI, J. et R. MERCER (1991) : «Aligning Sentences in Parrallel Corpora», Proceedings of the 29th Annual Meeting of the Association for Computational Linguistic's, Berkeley, pp. 169-176.

DYMETMAN, M., ISABELLE P. et F. PERRAULT (1990): «A Symmetrical Approach to Parsing and Generation», Proceedings of the $13 \mathrm{th}$ International Conference on Computational Linguistics (COLING-90), Helsinki.

GALE, W. et K. CHURCH (1991): «A Program for Aligning Sentences in Bilingual Corpora», Proceedings of the 29th Annual Meeting of the Association for Computational Linguistics, Berkeley, pp. 177-184.

HARRIS, B. (1988) : «Are you Bi-Textual?», Language Technology, 7, p. 41.

HARRIS, B. (1988) : «Bi-text, a New Concept in Translation Theory», Language Monthly, 54, pp. 8-10.

ISABELLE, P., DYMETMAN, M. et E. MACKLOVITCH (1988): «CRITTER: a Translation System for Agricultural Market Reports», Proceedings of the 12th International Conference on Computational Linguistics (COLING-88), Budapest.

MACKLOVITCH, E. (1989) : «An Off-the-shelf Workstation for Translators», D. Hammond (Ed.), Proceedings of the 30th Annual Conference of the ATA, Washington DC, October 11-14, pp. 489-498. (Également disponible en français sous forme de rapport technique du CCRIT.)

MACKLOVITCH, E. (1991): A Second Version of the CWARC's Workstation for Translators, rapport technique du CCRIT.

SATO, S. et M. NAGAO (1990): «Toward Memory-Based Translation», Proceedings of COLING-90, pp. $247-252$.

SIMARD, M., FOSTER, G. et P. ISABELLE (à parâtre): Using Cognates to Align Sentences in Bilingual Corpora.

Socioscope Inc. (1986): Functional Specifications for a Translator's Workstation, rapport présenté au Centre canadien de recherche sur l'informatisation du travail, contrat 12SD.36902-5-0003.

\section{ANNEXE : EXEMPLES DE CONCORDANCES}

Les pages qui suivent présentent des exemples de concordances monolingues et bilingues. Ces concordances ont toutes été effectuées sur le Journal des débats de la Chambre des Communes.

Dans les concordances, les segments sont précédés de leur date d'apparition dans le Journal des débats. Les segments en langue source sont préfixés par l'indicateur «S:», alors que ceux qui sont des traductions sont préfixés par l'indicateur «T:».

Nous donnons d'abord un exemple d'utilisation de cet outil traditionnel que constitue la concordance monolingue. Cet outil permet au langagier de se faire rapidement une idée de l'utilisation qui est faite, dans une banque de textes, d'un mot (ou d'une suite de mots) particulier. Le terme countervail n'est guère connu que des spécialistes du commerce international, mais on voit que la concordance nous donne très rapidement une idée de son emploi. Deux journées de débats (avril 1986) nous ont fourni suffisamment d'exemples. 
La concordance bilingue est quant à elle un outil entièrement nouveau, rendu possible par le développement de techniques de création automatique de bi-textes. Le premier exemple que nous donnons reprend le terme countervail, en produisant la traduction de chacun de ses contextes d'apparition. On remarquera que les traducteurs font preuve d'une certaine hésitation entre droit compensatoire et droit compensateur comme traduction de countervail.

Les deux autres exemples concernent plutôt des expressions de la langue générale : cartes sur table en français, et add insult to injury en anglais. Ces expressions sont plus rares, et la machine a parcouru environ 35 millions de mots pour nous ramener ces quelques pages d'exemples. Avec lin logiciel approprié, ceci peut se faire de façon quasi instantanée. L'examen des traductions révèle une richesse insoupçonnée. Si à l'occasion certaines traductions paraissent banales, le lecteur conviendra qu'il peut rapidement identifier beaucoup de solutions intéressantes, qu'il n'aurait sans doute jamais trouvées dans les dictionnaires disponibles.

\section{Concordance monolingue sur «countervail»}

860421

$>$ S: Would he inform us what action the Government is proposing to take to protect Canadian steel companies, specifically

Ipsco, from countervail

$860428:: ;::::::::$

$>\mathrm{S}$ : What is the point of having a general trade agreement with the United States if the result of it will be that United States will still be able to impose countervail

$>$ S: One of the strongest weapons the U.S. has been using to restrict entry of foreign goods onto its market has been

countervail

$>\mathrm{S}$ : Will the Government assure us that any free trade agreement entered into with the U.S. will contain clauses explicitly banning the use of countervail $>S:>\mid$ Right Hon. | Joe Clark

(Secretary of State for External | I Affairs):

I Mr. Speaker,

countervail

$>\mathrm{T}:>\mid \mathrm{Mr}$. Jacques Guilbault (Saint-Jacques):

IMr. Speaker, the Government cannot refuse to shed

light on its position on the use of countervail

$>\mathrm{T}:>$ Again I want to ask the Secretary of State for

External Affairs: Is the position of the

Canadian Government such as

to let the United States levy

countervail

$>\mathrm{T}:>$ | Right Hon. Joe Clark (Secretary of State for External | | Affairs): | Mr. Speaker, questions

related to

$>$ S: To let the U.S. continue threats of

countervail

countervail

$>S:$ Is the Minister aware of the letter President Reagan sent to the Senators whose support he got last week in which he made a commitment to countervail $>S$ : Is the Minister prepared to say to the President what he has just said to the House, that countervail duties which the U.S. Commerce Department recommended against certain Canadian steel products?

ing duties on our exports in areas such as lumber, and fish, and even specialty steel?

duties.

duties against Canadian goods?

is very much at the heart of these negotiations.

ing duties by the United States.

ing duties in retaliation under a free trade agreement with our country?

ing action are an essential aspect of the negotiations. ing duties while the talks are going on is like permitting war to continue while you are discussing a ceasefire.

ing action?

ing action is at the heart of negotiations and should not be used while the talks are taking place? 
$>$ S:>| Mr. Steven W. Langdon (Essex | |Windsor): I |Mr. Speaker, given that these threats will continue, will the Government protest to President Reagan and attempt to get a clear commitment from him that threats of countervail ing action will not be made against our producers during the long period of negotiations?

\section{Concordance bilingue sur «countervail»}

860421

S: Would he inform us what action the Government is proposing to take to protect Canadian steel companies, specifically Ipsco, from countervail duties which the U.S. Commerce Department recommended against certain Canadian steel products?

\section{$860428 * * * * * * * * * * * *$}

$\mathrm{S}$ : What is the point of having a general trade agreement with the United States if the result of it will be that the United States will still be able to impose countervailing duties on our exports in areas such as lumber, and fish, and even specialty steel?

S: One of the strongest weapons the U.S. has been using to restrict entry of foreign goods onto its market has been countervail duties.

S: Will the Government assure us that any free trade agreement entered into with the U.S. will contain clauses explicitly banning the use of countervail duties against Canadian goods?

S:> Right Hon. | Joe Clark (Secretary of State for External || Affairs) : | Mr. Speaker, countervail is very much at the heart of these negotiations.

T:>1 Mr. Jacques Guilbautt (Saint-Jacques): I Mr. Speaker, the Government cannot refuse to shed light on its position on the use of countervailing duties by the United States.

$T:>$ Again I want to ask the Secretary of State for External Affairs: Is the position of the Canadian Government such as to let the United States levy countervailing duties in retaliation under a free trade agreement with our country?

T:>l Right Hon. Joe Clark (Secretary of State for External || Affairs) : | Mr. Speaker, questions related to countervailing action are an essential aspect of the negotiations.

S: To let the U.S. continue threats of countervailing duties while the talks are going on is like permitting war to continue while you are discussing a ceasefire.

$S:$ Is the Minister aware of the letter President Reagan sent to the Senators whose support he got last week in which he made a commitment to countervailing actions?
$\mathrm{T}$ : Pourrait-il nous dire ce que le gouvemement a l'intention de faire pour protéger les sociétés sidérurgiques canadiennes, plus précisément Ipsco contre les droits compensatoires que le département américain du Commerce a demandé de prendre contre certains produits sidérurgiques canadiens?

$\mathrm{T}$ : $\grave{A}$ quoi sert-il de conclure un accord commercial général avec les États-Unis si ces derniers vont pouvoir continuer à imposer des droits compensateurs sur nos exportations dans certains secteurs tels que le bois d'cuvre et le poisson ou même certains aciers ?

T: Les droits compensateurs constituent l'une des principales armes que les États-Unis ont utilisées pour restreindre les importations de produits étrangers sur leur marché.

T: Le gouvernement peut-il nous assurer que si nous concluons une entente de libre-échange avec les États-Unis, des clauses spéciales seront prévues pour interdire l'application de droits compensateurs à l'égard des marchandises canadiennes?

T:>| Le très hon. Joe Clark (secrétaire d'État aux affaires | | extérieures): | Monsieur le Président, les questions de kcountervaiN sont au cour des négociations.

S:>| M. Jacques Guilbault (Saint-Jacques) :| Monsieur le Président, le gouvernement ne peut pas refuser de clarifier sa position sur l'utilisation des droits compensateurs par les États-Unis.

$S:>$ Je veux demander encore une fois au secrétaire d'État aux Affaires extérieures: Est-ce la position du gouvernement canadien de laisser les États-Unis utiliser les droits compensateurs en guise de représailles dans un accord de libre-échange avec notre pays?

T:>l Le très hon. Joe Clark (secrétaire d'État aux affaires | | extérieures): | Monsieur le Président, la question des droits compensateurs est au cour de ces négociations.

T: Permettre aux États-Unis de nous menacer continuellement d'imposer des droits compensateurs pendant que les entretiens se déroulent, c'est un peu comme laisser la guerre se poursuivre pendant que l'on discute d'un cessez-le-feu.

T: Le ministre est-il au courant de la lettre que le président Reagan a envoyée aux sénateurs qui l'on soutenu la semaine dernière et où il promet d'imposer des droits compensateurs? 
S: Is the Minister prepared to say to the President what he has just said to the House, that countervailing action is at the heart of negotiations and should not be used while the talks are taking place?

S:I Mr. Steven W. Langdon (Essex-Windsor): IIIMr. Speaker, given that these threats will continue, will the Government protest to President Reagan and attempt to get a clear commitment from him that threats of countervailing action will not be made against our producers during the long period of negotiations?
T: Le ministre est-il disposé à dire au président ce qu'il vient de dire à la Chambre, à savoir que ces droits sont au cœur des négociations et qu'ils ne doivent pas être imposés pendant la durée des entretiens?

$T:>1$ M. Steven W. Langdon (Essex-Windsor): | Monsieur le Président, étant donné que ces menaces vont continuer, le gouvernement va-t-il protester auprès du président Reagan et obtenir sa promesse que les États-Unis ne menaceront pas d'imposer des droits compensateurs à nos producteurs pendant toute la durée des négociations?

\section{Concordance bilingue sur «cartes sur table»}

$860506 * * * * * * * * * * * *$

T: Mais maintenant les cartes sont sur la table, et les contribuables de l'Ontario et d'ailleurs dégustent.

860522

T: Peut-il jouer cartes sur table?

$860523 * * * * * * * * * * * *$

T: Le gouvernement est-il prêt à mettre cartes sur table et à faire savoir aux Canadiens quelles sont les options qu'il examine?

$860603 * * * * * * * * * * * * * *$

T:> En étant trop généreux au début - et je peux énumérer toute une série de largesses consenties par le gouvernement sans exiger quoi que ce soit en retour -, nous nous sommes retrouvés en définitive quand les véritables négociations ont été entamées il y a quelques semaines, avec nos cartes sur table.

$860609 * * * * * * * * * * * * *$

T:> Pour ce qui est de négociations cartes sur table, je ne peux m'empêcher de faire remarquer que les preuves arrivent de tous bords et de tous côtés.

$870209 * * * * * * * * * * * * *$

T: S'il a pris une décision fondamentale à l'égard de sa stratégie industrielle, qu'il mette cartes sur table afin que nous tenions un débat sur la stratégie industrielle.

$870305 * * * * * * * * * * * *$

T: Tout d'abord, il doit jouer cartes sur table et dire que la Commission du blé a recommandé une diminution de 20 p. 100 , si c'est le cas.

$\mathrm{T}:>$ Je crois qu'en outre, les agriculteurs souhaitent que le gouvernement joue cartes sur table et dise exactement quel sera le prix de base des grains.

$870316 * * * * * * * * * * * * * *$

T: Il devrait jouer cartes sur table avec les Canadiens et nous dire ce qu'il fera pour les villes tributaires d'une industrie unique.

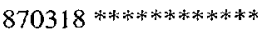

$\mathrm{T}:>\mid$ M. John McDermid (secrétaire parlementaire de la ministre du | | Commerce extérieur) : I Monsieur le Président, qu'il est amusant d'entendre les libéraux dire d'une part qu'il faut abattre toutes nos cartes sur la table, quand leur chef affirme d'autre part que nous avons montré trop de nos cartes dans les négociations.
$S$ : Now the cards are on the table. Ontario and other taxpayers are getting it in the neck.

S: Will he come clean with the Canadian people?

S: Is the Government ready to lay its cards on the table and inform Canadians about what options it is considering?

S:> By giving away so much at the front end - and I can go through the litany of freebies that were given away by this Government with nothing in return - when it came to the real bargaining that started a few weeks ago we had already put all our chips on the table.

S $>$ With respect to clean launch, I just cannot resist saying, the evidence builds up from every quarter.

$\mathrm{S}$ : If the Government was making a fundamental decision about industrial strategy, we should put all the cards on the table and have a debate about industrial strategy.

S: But first the Grovernment has to come clean and say that the Wheat Board has recommended a reduction of 20 per cent, if that is what it is.

$S$ : I believe there is concern as well with the Government coming clean and outlining exactly what the initial grain price will be.

S: He should come clean with Canadiens and tell us what he will do for single-industry towns.

S:>| Mr. John McDermid (Parliamentary - Secretary to Minister for | International Trade): | Mr. Speaker, it is interesting to hear the Liberals say on the one hand that we must lay all our cards on the table while on the other hand their Leader says we have shown too many cards in the negotiations. 
$870324 * * * * * * * * * * * * * *$

T: Il devrait jouer cartes sur table avec les Canadiens.

\section{$870427 * * * * * * * * * * * *$}

$\mathrm{T}$ : The Government must put its cards on the table. $870505 * * * * * * * * * * * *$

T: On ne peut pas jouer cartes sur table dans une partie de poker et on ne peut pas divulguer les détails au grand jour comme le souhaitent sans doute les Canadiens pour savoir ce qui se passe dans ce genre de négociations.

$870601 * * * * * * * * * * * * * * *$

T: Au moment même où l'on nous faisait la leçon sur l'importance qu'il y avait à jouer cartes sur table et à assurer une concurrence ouverte et équitable, nos exportations se ralentissaient quatre fois plus vite que le marché.

$870817 * * * * * * * * * * * * *$

T:> Il semble aujourd'hui avoir une nouvelle idée. Puisque le gouvernement est déterminé à suivre les conseils, notamment du premier ministre libéral du Québec, en faisant tout son possible pour négocier un accord commercial, il nous demande d'agir de façon à jouer cartes sur table, ce qui servirait les intérêts des Américains au point d'anéantir totalement notre position de négociation.

$870818 * * * * * * * * * * * * *$

T: Si c'est le cas, mettons cartes sur table et faisons savoir que le gouvernement ne compte plus respecter ses engagements internationaux.

$871009 * * * * * * * * * * * *$

T: Que le gouvernement joue cartes sur table avec les Canadiens, qu'il leur dise ce qu'il a fait et pourquoi il l'a fait.

$871201 * * * * * * * * * * * * *$

T: Il a mis cartes sur table.

$871214 * * * * * * * * * * * *$

T:> Le gouvernement doit mettre cartes sur table.

$871215 * * * * * * * * * * * * *$

S: C'est ce que le public canadien désire savoir, mettez-donc les cartes sur table!

$880321 * * * * * * * * * * * * *$

T: J'ai écrit que nous avions été complaisants assez longtemps et qu'il fallait maintenant mettre cartes sur table pour dire que le temps d'agir était venu.

T: Par conséquent, commc je l'ai fait observer dans ma lettre au premier ministre, ce dernier a le beau rôle et j'ose espérer qu'il mettra cartes sur table.

$880816 * * * * * * * * * * * *$

T: De toute évidence, cet amendement oblige le gouvernement à mettre cartes sur table et à dévoiler son intention d'ouvrir nos services de santé aux profiteurs des États-Unis.
S: It should present Canadians with the straight goods.

S: Le gouvernement doit mettre ses cartes sur la table.

S: One cannot lay one's cards on the table in a poker game, and we cannot lay them on the table perhaps the way Canadians would prefer so they would know what is going on in negotiations like this.

S: At the very time when we were being lectured on the virtues of the level playing field and the need to ensure open and fair competition, our exports were declining four times faster than the rate at which the market was shrinking.

S:> Now he seems to have a new proposal and that new proposal is that since the Government is determined to follow the advice of, among, others the Liberal Premier of the Province of Quebec, by proceeding with the best efforts attempt to negotiate a trade arrangement, he is trying to ask us to act in a way that would put the Canadian case on the table for the advantage of the Americans in a way that would destroy the Canadian case.

S: If that is the case, let us get it on the table and say that the Government no longer agrees with its international commitments.

S: Let the Government be straight with Canadians as to why it did what he did.

S: He has put his facts on the table.

$\mathrm{S}:>$ The Government must be clear for the Canadian people.

$\mathrm{T}$ : That is what Canadians wants to know. Put your cards on the table!

S: I wrote that we have been nice guys long enough, we must lay it on the line and say that something must be done.

$\mathrm{S}$ : Therefore, as I pointed out in my letter to the Prime Minister, on this problem he is on the side of the angels and I would hope that he will certainly lay it on the line.

$\mathrm{S}$ : It is clear that this amendment calls the Government's bluff and exposes its intention to open our health services to the profiteers from the United States. 
$881220 * * * * * * * * * * * * *$

T: Nous affirmons qu'ils ne peuvent se prononcer sur cette question que si l'on met cartes sur table et que si la population canadienne dispose de tous les moyens voulus pour comprendre ce qui se passe.

$881223 * * * * * * * * * * * * *$

$T$ : Est-ce que vous vous lanceriez dans une grosse entreprise commerciale avec une autre société sans d'abord vous assurer qu'on joue cartes sur table, que le crédit de votre futur partenaire est bon et que tous vos associés sont au courant de la situation?
S: We say that that judgment cannot be made unless there is full and open disclosure and full opportunity for the people of Canada to understand what is going on.

S: Would you venture into a major business deal with another company without first making sure that all the cards were on the table and that credit was good at the bank and that all your business partners knew what was going on?

Concordance bilingue sur «add insult to injury»

$870128 * * * * * * * * * * * * *$

S:> To add further insult to injury, just last week the Minister of Energy, Mines and Resources (Mr. Masse), in a speech to the Montreal Chamber of Commerce, was quoted as saying the following: $870213 * * * * * * *$

$S:$ It adds insult to injury to receive the paltry sum of $\$ 350$ million for such a lucrative company, so valuable to the people of Canada.

$870316 * * * * * * * * * * * *$

$\mathrm{S}:>\mid$ Mr. Tobin: | Given that the Prime Minister added insult to injury this morning, saying that only the Government of Canada has the power to make foreign treaties, and given that U.S. trade negotiator Peter Murphy said this morning -

$870330 * * * * * * * * * * * *$

S: It adds insult to injury to receive only a paltry $\$ 350$ million for such a lucrative company so valuable to the people of Canada.

$870413 * * * * * * * * * * * * * *$

S: To add insult to injury, 25 other Correctional Service projects throughout the country have been cancelled.

$870430 * * * * * * * * * * * *$

$T:>I$ fail to understand why the Government, which had already announced a few days before that grain producers could expect an 18 per cent cut in prices, then chose to add insult to injury by announcing it would no longer guarantec loans that were in arrears.

\section{$\{70505 * * * * * * * * * * * * *$}

$S:>$ To add insult to injury, the petitions directed to "Concerned Canadians" contained a letter that accurately described the Hon. Member's Party Leader, the Right Hon. Member for Vancouver Quadra (Mr. Turner), as being the proponent of the legislation this Government is attempting to change.

$870507 * * * * * * * * * * * *$

S:> Finally, to add insult to injury, there is the UHot$100 \backslash$ as it is called, "A quick guide to what the feds are doing for you".
$\mathrm{T}:>$ Et pour comble d'outrage, le ministre de l'Énergie, des Mines et des Ressources (M. Masse) aurait dit ce qui suit la semaine dernière dans une allocution à la Chambre de Commerce de Montréal :

T: Il est scandaleux de se contenter de 350 millions pour une entreprise aussi lucrative et aussi précieuse pour les Canadiens.

$\mathrm{T}:>\mid \mathrm{M}$. Tobin: | Étant donné que le premier ministre a eu l'aplomb, ce matin, de déclarer que seul le gouvernement canadien pouvait conclure des traités avec les pays étrangers et que le négociateur commercial des États-Unis, Peter Murphy, a dit ce matin...

T: C'est vraiment le comble de ne recevoir que 350 millions de dollars pour une société aussi précieuse pour les Canadiens.

T: Le comble, c'est que 25 autres projets du Service correctionnel ailleurs au pays ont été supprimés.

S:> Donc, je ne peux pas comprendre qu'au même moment où le gouvernement annonce à quelques jours d'intervalle que le revenu des producteurs de grain sera inférieur de 18 p. 100 , on ajoute l'insulte à l'injure en leur disant à part cela qu'on ne garantira plus les prêts qui étaient en retard.

T:> Pour porter l'injustice à son comble, on a joint aux pétitions une lettre dans laquelle on présentait le chef du parti du député, l'honorable député de Vancouver Quadra (M. Turner), comme le parrain de la loi que le gouvemement souhaite modifier.

T:> Enfin, pour porter l'insulte à son comble, le gouvernement a publié \Palmarès $\backslash$ ou «Guide éclair des programmes et services jeunesse du gouvernement fédéral ». 
S: To add insult to injury, the Government has cut out the housing labour component which used to be available from Canada Employment and Immigration centres but is now absent under the Canadian Jobs Strategy.

$870512 * * * * * * * * * * * *$

S:> To add insuit to injury, the Government has also declared that the appellant be reported until the Federal Court has considered the appeal.

$870519 * * * * * * * * * * * * *$

S: They were told they would no longer have a job at Miracle Mart and, to add insult to injury, were told they would not be entitled to collect unemployment insurance.

$870605 * * * * * * * * * * * * *$

$\mathrm{S}$ : That adds insult to injury as far as the victims are concerned.

$870623 * * * * * * * * * * * *$

S: However, if committed in private, there had to be in addition an intent to insult or offend.

S:> In addition to being unable to collect unemployment insurance, the Government, adding insult to injury, insists that when they go to work they pay unemployment insurance premiums.

$870629 * * * * * * * * * * * * *$

$\mathrm{S}$ : Local markets for maritime producers will be swallowed by western imports - I am speaking of everything from Quebec west - and to add insult to injury, those western imports will be subsidized by transportation subsidies.

$870630 * * * * * * * * * * * *$

S:> This year's allocation of salmon was set below the minimum needed for many trollers to survive. Now, to add insult to injury, trollers are being shut down before they have been able to catch even this quota.

$870827 * * * * * * * * * * * * *$

S: To add insult to injury, the Prime Minister sent the weak and meek Member of Parliament from Halifax in the spring to announce refitting programs which would begin in November, 1987.

$870831 * * * * * * * * * * * * * *$

S:> To add insult to injury, we have a Minister who has the nerve to say, "Don't worry, you guys over there, the Inter-Church Committee, we will not prosecute you.

$871103 * * * * * * * * * * * *$

T: They want action and they want it now. Mr. Speaker, to add insult to insult, the Minister goes so far as to inform us he distributed this document as a courtesy.

$860423 * * * * * * * * * * * * * * *$

S: To add insult to injury we in Nova Scotia - a have-not province - are being discriminated against by the Government.
T: Comme si cela ne suffisait pas, le gouvernement a supprimé les possibilités d'emploi dans le bâtiment qu'offraient les Centres d'emploi et d'immigration du Canada mais qui n'existent plus dans le cadre de la Planification de l'emploi.

T:> Le comble, c'est que le gouvernement a également déclaré que celui qui porte sa cause en appel sera expulsé en attendant que la Cour fédérale étudie l'affaire.

T: On leur a dit qu'elles n'auraient plus d'emploi à Miracle Mart et, pour les achever, qu'elles n'auraient pas le droit de bénéficier de l'assurance-chômage.

T: Ce serait trahir à nouveau des victimes déjà bafouées.

S: Mais, lorsqu'il s'agissait d'un endroit privé, il devait $y$ avoir en plus une intention d'insulter ou d'offenser.

T:> Non seulement n'ont-ils pas pu toucher de prestations d'assurance-chômage, mais le gouvernement, comble d'insulte, a insisté pour qu'ils retournent au travail et paient des primes d'assurance-chômage.

T: Les marchés locaux des producteurs des Maritimes seront submergés par les importations de l'Ouest, je veux dire de toutes les régions à l'ouest du Québec, et, ce qui est encore pire, ces importations bénéficieront des subventions au transport.

T:> Après avoir fixê le volume des prises de saumon à un niveau inférieur à celui qui permettrait à de nombreux exploitants de chalutiers de survivre, il a encore ajouté à cette insulte en leur ordonnant de cesser toute activité avant même d'avoir atteint leur contingent.

T: Le comble, c'est que le premier ministre a envoyé le faible et timoré député de Halifax annoncer le printemps dernier des programmes de radoubage qui débuteraient en novembre 1987.

T:> Pour ajouter l'insulte à l'injure, le ministre a le culot de dire: «Ne vous tracassez pas, vous les membres du comité inter-églises, nous ne vous poursuivrons pas en justice.»

S: Non seulement, monsieur le Président, tous les francophones du Canada sont insultés de l'attitude du ministre et du gouvernement conservateur, mais il prend la peine de nous dire qu'il a distribué son document par courtoisie.

T:> Pour ajouter l'insulte à l'injure, nous, en NouvelleÉcosse - une province défavorisée - sommes objet de discrimination de la part du gouvernement. 
$860526 * * * * * * * * * * * *$

$\mathrm{S}$ : It has added insult to injury as far as the offshore is concerned.

860603

$S$ : Instead of addressing those doubts and talking about those serious concerns of not only Members of Parliament but hundreds of groups across the country, the Government retreated to a series of insults of which I could give you far too many examples today.
T: En ce qui concerne la prospection au large il a été doublement injuste.

T: Au lieu d'aborder de front ces appréhensions et de répondre aux très graves objections non seulement de certains députés, mais également de centaines de groupes d'un bout à l'autre du Canada, le gouvernement a choisi de lancer à la tête de l'Opposition toute une série d'injures dont je pourrais vous fournir aujourd'hui de trop nombreux exemples. 